\section{Diffuse intramural duodenal hematoma complicated by lethal necrotizing pancreatitis after endoscopic duodenal biopsy}

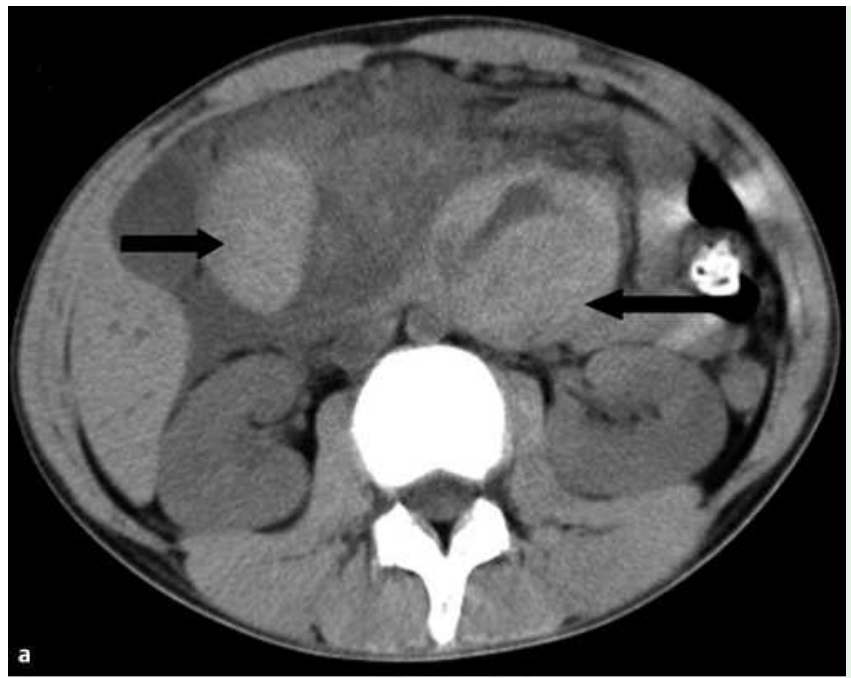

Fig. 1 Both the abdominal computed tomography images, without contrast (a) and with contrast (b), disclosed diffuse intramural hematoma (arrows) of the duodenum, extending from the first portion to the fourth portion.

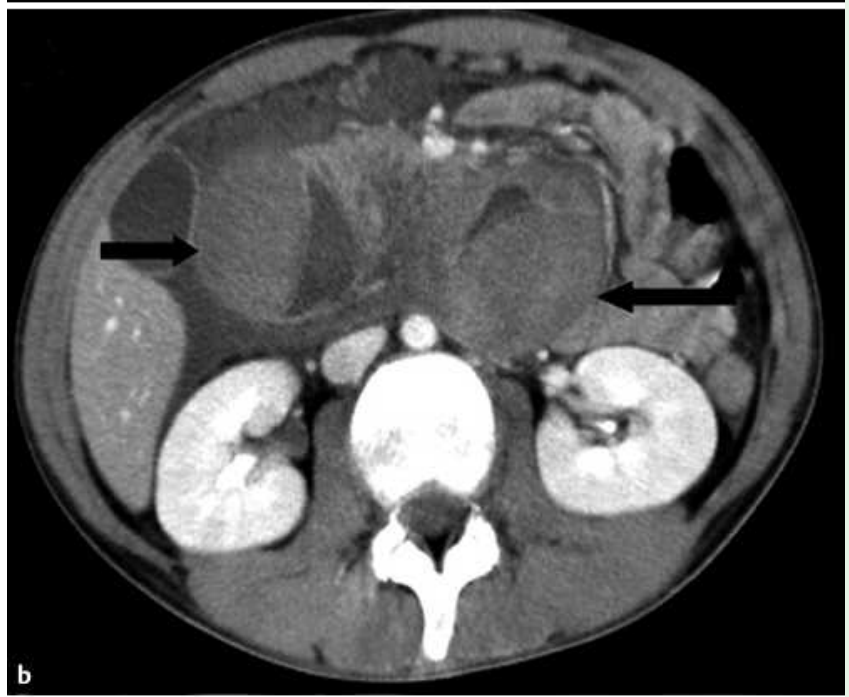

A 17-year-old boy with Hodgkin's lymphoma had been treated with autologous peripheral blood stem cell transplantation. He had evident hematological deficits, with thrombocytopenia $(56000 \times$ $10^{9} / \mathrm{L}$ ), but with normal coagulation. Due to persistent epigastralgia, panendoscopy was conducted in order to take a gastric biopsy and a duodenal biopsy to exclude graft-versus-host disease after the platelet transfusion. One day later, the boy suffered abdominal pain, fullness, and vomiting. Physical examination demonstrated diffuse tenderness over the upper abdominal quadrant. The hematocrit dropped from $30.8 \%$ to $22.6 \%$. Abdominal computed tomography without $(\bullet$ Fig. 1 a) and with contrast ( $\bullet$ Fig. 1 b) disclosed a diffuse intramural hematoma of the duodenum, extending from the first portion to the fourth portion. This diffuse duodenal intramural hematoma resulted in severe necrotizing pancreatitis with acute respiratory distress syndrome and septic shock. The patient died 2 weeks later.

Duodenal intramural hematoma is a rare condition, and can sporadically be seen in patients with trauma, hematological deficits, or pancreatic cancer, or in those using anticoagulants [1-4]. Iatrogenic trauma caused by either endoscopic duodenal biopsy or therapeutic injection to a bleeding ulcer can also lead to duodenal

intramural hematoma, but is usually self-limited without lethal outcome $[4,5]$. However, in our patient the duodenal biopsy induced a lethal hematoma: it was diffusely extended from the first to the fourth portion and thus obliterated the pancreatic drainage, leading to severe necrotizing pancreatitis. Evacuation of the hematoma by surgery was limited by the hematological deficits in this case.

Despite the correction of the platelet counts and normal coagulation parameters, this case shows that the risk of mortality from duodenal biopsy still exists. Caution dictates that duodenal biopsy should be avoided during panendoscopy in patients in whom the possibility of surgery is limited by hematological deficits.

\title{
Endoscopy_UCTN_Code_CPL_1AH_2AB
}

\section{P. S. Chen, H. C. Cheng, B. S. Sheu}

Department of Internal Medicine, National Cheng Kung University Hospital, Tainan,

Taiwan, Republic of China

\section{References}

1 Ikeda T, Koshinaga T, Inoue $M$ et al. Traumatic intramural hematoma of duodenum with thrombasthenia in childhood. Pediatr Int 2007; 49: 668-671

2 Chou AL, Tseng KC, Hsieh YH et al. Intramural duodenal hematoma as a complication of pancreatic cancer. Endoscopy 2007; 39: E107-E108

3 Wang JY, Ma CJ, Tsai HL et al. Intramural duodenal hematoma and hemoperitoneum in anticoagulant therapy following upper gastrointestinal endoscopy. Med Princ Pract 2006; 15: $453-455$

4 Sugai K, Kajiwara E, Mochizuki Yet al. Intramural duodenal hematoma after endoscopic therapy for a bleeding duodenal ulcer in a patient with liver cirrhosis. Intern Med 2005; 44: 954-957

5 Sgouros SN, Karamanolis G, Papadopoulou E et al. Postbiopsy intramural hematoma of the duodenum in an adult with Noonan's syndrome. J Gastroenterol Hepatol 2004; 19: $1217-1219$

Bibliography

DoI $10.1055 / s-2007-995736$

Endoscopy 2008; 40: E143

(c) Georg Thieme Verlag KG Stuttgart · New York . ISSN 0013-726X

\section{Corresponding author}

\section{B. S. Sheu, MD}

Department of Internal Medicine

National Cheng Kung University Hospital

138 Sheng Li Road

Tainan

Taiwan 70428

Republic of China

Fax: +886-6-2370941

sheubs@mail.ncku.edu.tw 